- The body of evidence available on the efficacy and safety of water fluoridation was of lower quantity and quality than had previously been reported.

- The best available evidence (from studies that met inclusion criteria) suggests that fluoridation of drinking water supplies reduces caries prevalence but is associated with dental fluorosis.

- The balance of the evidence did not show an association between any fractures and water fluoridation.

- No associations between water fluoridation and human cancer were found.

\title{
The York Review - A systematic review of public water fluoridation: a commentary
}

\author{
E. T. Treasure, ${ }^{1}$ I. G. Chestnutt, ${ }^{2}$ P. Whiting, ${ }^{3}$ M. McDonagh, ${ }^{4}$ P. Wilson ${ }^{5}$ and J. Kleijnen ${ }^{6}$
}

Following the election of the British government in May 1997, a policy document outlining proposals to improve the health of the nation was published. This document placed a major emphasis on reducing inequalities in health and recognised the continuing poor state of oral health in deprived communities. However, whilst acknowledging the benefits of water fluoridation as a caries preventive measure, the Department of Health suggested that most of the research had been carried out some years ago and furthermore, recognised that strong views were held both for and against fluoridation.

As a result of this document, ${ }^{1}$ the Chief Medical Officer commissioned a review to investigate the efficacy and safety of water fluoridation and the contract for the review was awarded to the NHS Centre for Reviews and Dissemination at the University of York (CRD). This centre has an international reputation for conducting systematic reviews and over the last seven years has produced 73 reports on an array of health-related topics. Due to the specialist nature of the fluoridation review, CRD invited the Dental Public Health Unit at the University of Wales College of Medicine to collaborate on the research, a unit with which York had worked previously.

${ }^{1}$ Professor, Dental Public Health Unit, University of Wales College of Medicine, ${ }^{2}$ Senior Lecturer, Dental Public Health Unit, University of Wales College of Medicine, Cardiff, Wales, ${ }^{3}$ Research Fellow, NHS Centre for Reviews and Dissemination, University of York, ${ }^{4}$ Investigator, Oregon Evidence Based Practice Center, Research Associate, Kaiser Permanente Center for Health Research, Portland, Oregon USA, ${ }^{5}$ Research Fellow, NHS Centre for Reviews and Dissemination, University of York, ${ }^{6}$ Director, NHS Centre for Reviews and Dissemination, University of York, York, England

Correspondence to: Professor E. T. Treasure, Department of Dental Health and Development, The Dental School, Heath Park, Cardiff, CF14 4XY, UK.

Email:Treasureet@cardiff.ac.uk

\section{Refereed Paper}

Received 14.02.01; Accepted 13.09.01

( ) British Dental Journal 2002; 192: 495-497
The review has now been published in two forms, firstly in an article in the British Medical Journal ${ }^{2}$ and secondly the full version of the published report by $\mathrm{CRD}^{3}$ which is also available on the Web (http://www. york.ac.uk/inst/crd/fluorid.htm). Since it was first announced, this review has attracted considerable interest. The purpose of this commentary is:

- to outline the objectives of the review

- to briefly highlight the findings of the review

- to discuss aspects of the review methodology, in particular the criteria for the inclusion of studies

- to highlight shortcomings of current evidence identified by the review

\section{OBJECTIVES OF THE REVIEW}

Five objectives were identified:

- What are the effects of fluoridation of public water supplies on the prevalence of dental caries?

- If water fluoridation is shown to have beneficial effects, what is the effect over and above that offered by the use of alternative interventions and strategies?

- Does water fluoridation result in a reduction of caries across social groups and between geographical locations, bringing equity?

- Does water fluoridation have negative effects, eg dental fluorosis (mottled teeth), bone fractures, cancer?

- Are there differences in the effects of natural and artificial water fluoridation?

\section{FINDINGS OF THE REVIEW}

Beneficial outcomes of water fluoridation Dental caries

The best available evidence suggests that fluoridation of drinking water supplies reduces caries prevalence, both as measured by the proportion of children who are caries free and by the mean dmft/DMFT score. The studies were of moderate quality, but of limited quantity. The degree to which caries is reduced, however, is not clear from the data available. The difference in the number of children found to be caries free in fluoridated and non-fluoridated areas ranged from $-5.0 \%$ to $64 \%$ with a median of $14.6 \%$ in the 19 included analyses. The mean difference of change in $\mathrm{dmft} / \mathrm{DMFT}$ was from 0.5 to 4.4 teeth with a median of 2.25 teeth. The best available evidence on stopping water fluoridation indicates that caries prevalence increases, approaching the level of the low fluoride group, suggesting a previous benefit of water fluoridation. Again, the studies were of moderate quality, but of limited quantity. As a result, the available data (from studies that met inclusion criteria) provide 
only modest to moderate confidence in the ability to answer the objective.

Overall, the quality of studies on the effectiveness of water fluoridation was lower than had been expected and a large number of studies were excluded. To be included in the review, studies on the effectiveness of fluoridation had to have a positive answer to the following questions:

- Are at least two populations compared?

- Are there different water fluoride concentrations in the two populations?

- Is it a prospective study design? (ie there were baseline and follow-up examinations)

- Is the change in fluoride status within the last year (three years for moderate quality papers)?

- Was at least one of the following outcome measures used: DMFT, dmft, deft, DMFS, dmfs, \% caries free teeth/subjects?

Any study which did not answer 'yes' to any of the above questions was excluded.

A large number of the investigations into the effect of water fluoridation on dental caries were not included in the review due to their cross-sectional design, and thus did not meet the inclusion criteria outlined above. All but two of the studies included were before-after studies, two of the included studies used prospective cohort designs. All before-after studies located by the search were included. The most serious defect of these studies was the lack of appropriate analysis. Many studies did not present an analysis at all, while others only carried out simple analyses without attempting to control for potentially confounding factors. While some of these studies were conducted in the 1940s and 50s, prior to the common use of such analyses, studies conducted much later also failed to use methods that were commonplace at the time of the study.

Another defect of many studies was the lack of any measure of variance for the estimates of decay presented. All but two studies that presented the proportion of caries-free children contained sufficient data to calculate standard errors. This was not possible for the studies that presented $\mathrm{dmft} / \mathrm{DMFT}$ scores. Only four of the eight studies using these data provided estimates of variance.

The differences where fluoride had been removed from the water were less clear. Three studies were unable to detect a difference while four studies found significant differences, ie that the removal of fluoride from the water led to a greater increase in caries in the previously fluoridated group compared with the unfluoridated group.

There was significant heterogeneity (differences in effect) between the studies. In this review, it was decided to try to investigate the heterogeneity using a multivariate, meta-regression analysis.
From the meta-regression analysis it is interesting to note the difference in what happens to the proportion of caries free and the mean DMFT/dmft with differing baseline levels of disease. At low baseline levels of caries as measured by the DMFT/dmft, children with a higher baseline DMFT/dmft had a greater reduction in DMFT/dmft. In contrast the increase in the number of caries free was greatest in those with a low baseline caries level as measured by the percentage caries free. At first sight this may appear to be contradictory but is more easily understood if it is thought about in terms of the distribution of DMFT/dmft. It is much easier to make a child caries free if they have a lower level of caries. The analysis showed that there was no difference in the mean difference in the change in $\mathrm{dmft} / \mathrm{DMFT}$ or percentage caries free in studies conducted before and after 1970 . This may suggest that water fluoridation may still be of benefit after the advent of fluoride toothpaste. It may be considered surprising that no difference was found comparing before 1970 and after 1970 given the reduction in caries prevalence reported over that time. Using the date of publication may not have been sufficiently sensitive to identify any change.

\section{Water fluoridation and social class}

Within the United Kingdom, dental caries is strongly associated with dental deprivation. The Acheson Report stated that water fluoridation should decrease inequalilties in dental caries between areas and between socio-economic groups ${ }^{4}$. The search for evidence found no longitudinal studies that addressed this issue. It was decided, therefore, to examine cross-sectional studies but to limit these to those from the United Kingdom. This was because of the likelihood of there being problems in comparing studies from different countries where different measures of social class have been used.

There appears to be some evidence that water fluoridation reduces the inequalities in dental health across social classes in 5and 12-year-olds, using the $\mathrm{dmft} / \mathrm{DMFT}$ outcome measure. This effect was not seen in the proportion of caries-free children among 5-year-olds. The data for the effects in children of other ages did not show an effect. The small quantity of studies, differences between these studies and their low quality rating suggests caution in interpreting these results.

\section{ADVERSE OUTCOMES OF WATER FLUORIDATION}

The inclusion criteria for papers being assessed for adverse effects were less stringent than for those determining positive outcomes. Thus to be included the follow- ing criteria had to be fulfilled:

- at least two populations compared.

- different fluoride concentrations in the two populations.

\section{Dental fluorosis}

Fluorosis was the most widely and frequently studied of all the adverse effects considered. The fluorosis studies were largely cross-sectional designs, with a few before-after designs. Although numerous studies of fluorosis were included, all but one of the studies were of low quality. Observer bias may be of particular importance in studies assessing fluorosis. Efforts to control for potential confounding factors, or reducing potential observer bias, were uncommon.

As there may be some debate about the significance of a fluorosis score at the lowest level of each index being used to define a person as 'fluorosed', a second method of determining the percent 'fluorosed' was selected. This method describes the number of children having dental fluorosis that may cause 'aesthetic concern' and followed the method described by Hawley et al. ${ }^{5}$

With both methods of identifying the prevalence of fluorosis, a significant doseresponse relationship was identified through a weighted univariate regression analysis. The prevalence of fluorosis at a water fluoride level of $1.0 \mathrm{ppm}$ was estimated to be $48 \%$ and for fluorosis of aesthetic concern it was $12.5 \%$. At $0.1 \mathrm{ppm}$ the prevalence of fluorosis was found to be $15 \%$ and with fluorosis of aesthetic concern 6\%.

The multivariate analysis of fluorosis took into account variables that might have been contributing to the heterogeneity between studies. This analysis found a statistically significantly higher risk in children with permanent teeth, compared with those with primary teeth or both types of teeth. The multivariate analysis of fluorosis of aesthetic concern confirmed these findings. Interestingly, year of study, altitude and temperature were not found to be important in this analysis. Although these findings may be counterintuitive, it raises the need to re-examine the effect of altitude, temperature and year of study with fluorosis. Nine studies adjusted for possible confounding factors of other sources of fluoride but the results were mixed and no definite association between the other sources of fluoride studied and fluorosis were able to be identified.

\section{Bone fractures}

The evidence on bone fracture can be broken down into hip fracture and other sites because there are more studies on hip fracture than any other site. Using a qualitative method of analysis (ie visually examining forest plots), there is no clear association of 
hip fracture with water fluoridation. The evidence on other fractures is similar. Overall, the findings of studies of bone fracture effects showed small variations around the 'no effect' mark.

Outside of fluorosis, bone effects (not including bone cancers) were the most studied potential adverse effect. The validity of these studies was relatively low.

The studies included a variety of designs: prospective cohort studies, retrospective cohort studies, ecological studies, a case control study and one study that used a case control and ecological design. The studies also included different types of fractures and different age groups. Only some of the studies controlled for potential confounding factors. The relative risk with confidence intervals was calculated for each study. The heterogeneity between studies was too great to allow the data to be pooled. It was not possible to find any significant association between bone fracture or hip fracture and water fluoridation.

\section{Cancer}

The review concluded that no associations between cancer and water fluoridation were able to be detected.

Although the evidence regarding cancer was of a higher level than the other negative effects studies, the quality of the majority of studies was still relatively low. Results were presented for different types of cancers including all-site cancers, osteosarcoma, bone cancer, thyroid cancer and other site-specific cancers. A variety of measures were used to compare the cancer rates: standardised incidence rates, standardised mortality rates, crude rate ratios, mean differences in SMRs and mean differences of change in SMRs.

\section{Other adverse effects}

A total of 33 studies of the association of water fluoridation with other possible adverse effects were included in the review. The outcomes examined included Down's Syndrome, mortality, senile dementia, goitre and IQ levels. Overall, the included studies examining other possible negative effects provided insufficient evidence on any particular outcome to permit confident conclusions.

Interpreting the results of the other possible negative effects proved very difficult because of the small numbers of studies that met inclusion criteria on each specific outcome, the study designs used and poor study quality. A major weakness of these studies generally was the lack of control for any confounding factors, many of which were highlighted by the study authors. In the case of the Down's syndrome studies, for example, all six studies used designs that measured population rather than individual exposures to fluoridated water and, because of this, are particularly susceptible to confounding. If the populations being studied differed in respect to other factors that are associated with the outcome under investigation, then the outcome may differ between these populations leading to an apparent association with water fluoride level. A separate publication ${ }^{6}$ discusses these issues in detail.

\section{Differences in the effects of natural and artificial water fluoridation}

The assessment of natural versus artificial water fluoridation effects was greatly limited due to the lack of studies making this comparison. Very few studies included both natural and artificially fluoridated areas, and direct comparisons were not possible for most outcomes. No major differences were apparent in this review; however, the evidence was not adequate to make a conclusion regarding this objective.

\section{SUMMARY/CONCLUSIONS}

To our knowledge, the York review is the first systematic review to be undertaken on the subject of water fluoridation. It has undertaken a rigorous search of the published and unpublished literature of human epidemiological studies in all languages. The protocol was subject to external review as were all intermediate stages of the review. Throughout the review, progress and intermediate findings were published on the worldwide web.
The review was critical of the body of evidence that was identified. The authors were surprised by the small amount of work identified. In particular, there were very few studies that followed the same individuals longitudinally, there was lack of analysis of confounding variables and there was failure to undertake appropriate statistical analysis. If new schemes were to start, evaluation should include a robust study design that addressed these issues. One or a few good cohort studies with well-chosen objectives that adequately deal with relevant confounding factors and minimise bias in general would add considerably to the current knowledge about efficacy and safety of water fluoridation with regard to dental caries and fluorosis.

In a brief commentary such as this it is only possible to provide the briefest outline of the methodology and findings of a review of such a large body of literature. Interested readers are encouraged to pursue the main report of the review. From the evidence available, it can be concluded that fluoridation of public water supplies does prevent caries and is associated with fluorosis. The data presented suggest that the effect may be greater in those who have higher baseline levels of DMFT/dmft and this fact may be of use to those deciding health policy. It is imperative that future studies of water fluoridation, either new schemes or the discontinuation of fluoridation, should employ the highest methodological standards and appropriate data analysis.

1. Department of Health. Saving Lives: Our Healthier Nation. 1999; London, The Stationery Office.

2. McDonagh $M$, Whiting $P$, Wilson P etal. Systematic review of water fluoridation. BrMed J 2000; 321: 855-9.

3. McDonagh M, Whiting P, Bradley M et al. Asystematic review of public water fluoridation. 2000: York, Report number 18 University of York.

4. Acheson D. Independent Inquiry into Inequalities in Health. 1998; The Stationery Office.

5. Hawley G, Ellwood R, Davies R. Dental caries, fluorosis and the cosmetic implications of different TF scores in 14-year-old adolescents. Community Dent Health 1996: 13: 189-92.

6. Whiting P, McDonagh M, Kleijnen J. Association of Down's syndrome and water fluoride level: a systematic review of the evidence. In submission. 